\title{
Phylogenetic relationships of Coreanomecon (Papaveraceae: Papaveroideae), an endemic genus in Korea, using DNA sequences
}

\author{
Narae YUN and Sang-Hun $\mathrm{OH}^{*}$ \\ Department of Biology, Daejeon University, Daejeon 34520, Korea \\ (Received 11 November 2018; Revised 24 December 2018; Accepted 28 December 2018)
}

\begin{abstract}
Coreanomecon is a monotypic and endemic genus in Korea, distributed mainly in the southern regions. Coreanomecon is morphologically similar to Hylomecon by producing red latex, easily distinguished from Chelidonium, which produces yellow latex. Coreanomecon were merged into Hylomecon or Chelidonium depending on the authors. To understand the phylogenetic relationship of Coreanomecon, DNA sequences of chloroplast $r b c L$ and matK and nuclear Internal Transcribed Spacer (ITS) regions were determined from the species of Papaveroideae (Papaveraceae) in Korea and analyzed with the Maximum Parsimony and Bayesian methods. Phylogenetic analyses of Papaveroideae suggest that Coreanomecon is sister to the clade of Chelidonium and Stylophorum in the ITS data and that it is sister to Hylomecon in the chloroplast (cpDNA) data. A constraining analysis using the Shimodaira-Hasegawa test (S-H test) suggested that the ITS data do not reject the sister relationship of Coreanomecon and Hylomecon. The S-H test also suggested that the cpDNA data is compatible with the placement of Coreanomecon as a sister to the clade of Chelidonium and Stylophorum. Although the conflicting phylogenetic results may stem from insufficient phylogenetic signals, they may also be associated with hybridization between Hylomecon and an ancestor of Stylophorum and Chelidonium. The results of this study suggest that Coreanomecon is a distinct lineage as an endemic genus, supporting the morphological data.
\end{abstract}

Keywords: DNA barcoding, forensic botany, Papaveraceae, species identification

Papaveroideae (Papaveraceae) consists of 23 genera and approximately 240 species, characterized by having milky or colored latex sap, sepals fully enclosing the bud, actinomorphic corollas without spurs, numerous stamens, and a multicarpellate, usually unilocular gynoecium (Kadereit, 1993). Members of Papaveroideae are usually herbs and are distributed mainly in the Northern Hemisphere, extending into Central and South America (Kadereit, 1993). The subfamily was traditionally recognized as a separate family, Papaveraceae (Cronquist, 1988; Takhtajan, 1997; Kim, 2007), but Angiosperm Phylogeny Groups II, III, and IV (The Angiosperm Phylogeny Group, 2003, 2009, 2016) adopted a broad circumscription of Papaveraceae to include Fumariaceae and Pteridophyllaceae (Thorne, 1992; Mabberley, 2008). The more inclusive circumscription of Papaveraceae is supported by recent phylogenetic analyses using molecular and morphological data (Hoot et al., 1997, 2015; Wang et al., 2009), and it has been used in a broad range of recent literature (Ohwi, 1965; Zhang et al, 2008; Simpson, 2010; Hannan and Clark, 2011).

The diversity of Papaveroideae in Korea is relatively low with eight species in the four genera of Papaver L, Chelidonium L., Hylomecon Maxim., and Coreanomecon Nakai (Kim, 2007). The genus Papaver consists of approximately 80 species distributed in Central and southwestern Asia, with minor representatives in East Asia, Central and Southern Europe, and North Africa (Kadereit, 1993). Papaver somniferum L. is the best known species given its use in the production of opium, the dried latex from the fruits. Opium is the source of many important pharmaceutical drugs, including morphine, heroin, thebaine, codeine, and papaverine. Four species, including cultivated $P$. somniferum, are

\footnotetext{
*Author for correspondence: soh42@dju.kr
} 
distributed in Korea. Native species of Papaver in Korea, $P$. coreanum Nakai and $P$. amurense $(\mathrm{N}$. Busch) N. Busch ex Tolm., are distributed in the northern part of Korea, and their phylogenetic relationships have remained unclear. Owing to the importance in forensic botany to identify P. somniferum, the utility of DNA regions, in this case the nuclear Internal Transcribed Spacers (ITS) regions, chloroplast trnL-trnF regions and ISSR markers, were evaluated (Lee et al., 2010). These studies, however, did not include any native species of the Korean Papaver.

Chelidonium is a monotypic genus distributed in Europe and East Asia, and it is commonly distributed in Korea. Hylomecon is also a small genus with two species in East Asia, and $H$. vernalis Maxim. is commonly found in moist places in forests in Korea. Coreanomecon is a monotypic and endemic genus in Korea distributed mainly in the southern regions (Son et al., 2012). Coreanomecon is morphologically similar to Hylomecon by producing red latex, and it is easily distinguished from Chelidonium, which produces yellow latex. The taxonomic status of Coreanomecon has been controversial. Some authors merged Coreanomecon into Chelidonium (Ohwi, 1953), and others merged it into Hylomecon (Park, 1974; Kadereit, 1993). Palynological studies (Lee and Kim, 1984) and a cladistic analysis of the morphological characters (Kim et al. 1999) have suggested that Coreanomecon is distinct from Chelidonium and Hylomecon. The phylogenetic relationship of Coreanomecon according to molecular data have remained unclear.

DNA barcoding is a molecular technique which is used to identify a specimen with DNA sequences of short regions (Hebert et al., 2003; Kress et al., 2005; Xiang et al., 2011). The $r b c L$ and $m a t K$ regions in the chloroplast (cpDNA) genome are widely utilized in angiosperms (Hebert et al., 2004; CBOL Plant Working Group, 2009). The utility of other regions of cpDNA, such as trnH-psbA, atpF-atpH, and $p s b K-p s b I$, have been shown to be useful for increasing the resolution power (Lahaye et al., 2008; Kim et al., 2014). The ITS regions in nuclear ribosomal DNA have also been used to supplement cpDNA barcodes (Tripathi et al., 2013). DNA barcode regions have been primarily used for identifying unknown specimens at the species level. This type of molecular identification of a species is particular useful when the materials to be identified are fragmentary without having sufficient structures, such as flowers and fruits. Papaveroideae provides an excellent case, as it includes $P$. somniferum, often cultivated in Korea. A regional DNA barcode database for all known species is thus useful for not only applied fields, such as forensic botany, trade control at customs offices, conservation biology, and for the utilization of plant resources in bio-industries, but also for discovering cryptic species that are difficult to differentiate with morphological features and for reconstructing the phylogenetic relationships of the group of interest.

The objectives of the present study are (1) to infer the phylogenetic relationship of Coreanomecon, (2) to examine the implications of the molecular phylogeny on the evolution of the morphological characters of Coreanomecon, and (3) to assess the level of differentiation of the species and evaluate the resolution power of DNA barcodes to identify a species of Papaveroideae in Korea, with an emphasis on species of Papaver.

\section{Materials and Methods}

\section{Taxon sampling}

Analyses of Papaveroideae were conducted in three tiers. Analysis 1 includes Korean taxa only to evaluate the level of molecular differentiation among the Korean taxa. All but one species of Papaveroideae distributed in Korea were included in this study, with a total therefore of seven species in four genera (Table 1). Papaver amurense, distributed in North Korea and in northeast China, including Inner Mongolia, was not included because samples were not available. Two to six accessions per species were included to represent their distributional range, except for another North Korean species, $P$. coreanum Nakai, in which one sample from Baekdu Mt. was included. All materials for molecular studies were obtained from herbarium specimens deposited at the National Institute of Biological Resources (KB).

Analyses 2 and 3 expanded taxon sampling to infer the phylogenetic relationships of Coreanomecon and P. coreanum accurately. The chloroplast data from groups closely related to Coreanomecon, in this case Bocconia L., Dicranostigma Hook. f. \& Thomson, Glaucium Mill., Eomecon Hance, Macleaya R. Br., Sanguinaria L., and Stylophorum Nutt., were obtained from GenBank and added to our data set for Analysis 2. Analysis 3 included the nuclear ITS sequences of the tribe Chelidonieae, including Chelidonium and Hylomecon from foreign populations and those of the Papaver clade representing major subclades within the genus (Carolan et al., 2006). Analysis 3 was conducted to infer the placement of Coreanomecon and P. coreanum, as this has not been accomplished previously.

\section{Gene sampling}

Total DNA was extracted from dried leaves of herbarium specimens using a DNeasy Plant Mini Kit (QIAGEN, Hilden, 
Table 1. Taxa and voucher information included in this study.

\begin{tabular}{|c|c|}
\hline Taxa & Voucher \\
\hline \multirow[t]{4}{*}{$\begin{array}{l}\text { Chelidonium majus L. var. } \\
\text { asiaticum } \mathrm{H} \text {. Hara }\end{array}$} & $\begin{array}{l}\text { Gyeongsangbuk-do, Yecheon-gun, Hakgasan Mt., } 25 \text { May 2015, Kim, Kang, Park \& Bae HHU-2015-0297 } \\
\text { (KB 542414) }\end{array}$ \\
\hline & $\begin{array}{l}\text { Gyeongsangnam-do, Hamyang-gun, Gibaeksan Mt., } 5 \text { Jun 2015, Ko \& Park HNHM-KB-150128 (KB } \\
\text { 534818) }\end{array}$ \\
\hline & Jeollabuk-do, Sunchang-gun, Gyegwansan Mt., 30 Jul 2015, Im \& Park 150905328 (KB 542855) \\
\hline & Gangwon-do, Yeongwol-gun, Bongraesan Mt., 21 Apr 2012, Yoo \& Cheon 453815 (KB 453815) \\
\hline \multirow{5}{*}{$\begin{array}{l}\text { Coreanomecon hylomeconoides } \\
\text { Nakai }\end{array}$} & Jeollanam-do, Suncheon-si, Jogyesan Mt., 15 Jul 2013, Im \& Park Im81911 (KB 494404) \\
\hline & Jeollanam-do, Gurye-gun, Baekunsan Mt., 15 Jun 2012, Jang 363800 (KB 363800) \\
\hline & Jeollanam-do, Gurye-gun, Baekunsan Mt., 5 Jun 2013, Kim \& Kim seed3048 (KB 433738) \\
\hline & Chungcheongbuk-do, Jecheon-si, Woraksan Mt., 8 Sep 2007, Kim \& Kim 2007-2-012 (KB 531368) \\
\hline & Jeollanam-do, Suncheon-si, Jogyesan Mt., 13 Oct 2013, Lee 459419 (KB 459419) \\
\hline \multirow[t]{6}{*}{ Hylomecon vernalis Maxim. } & Gangwon-do, Hoengseong-gun, Eoreumgol, 1 May 2014, Kim, Lee \& Kim PH140147 (KB 483756) \\
\hline & Gangwon-do, Taebaek-si, Taebaeksan Mt., 6 Jun 2013, Kim \& Kim seed3059 (KB 433749) \\
\hline & Chungcheongbuk-do, Danyang-gun, Sobaeksan Mt., 9 May 2013, Lim, Nam \& Ryu celim061 (KB 464272) \\
\hline & Jeollabuk-do, Muju-gun, Jeoksangsan Mt., 18 May 2014, Kim \& Choi 90080 (KB 512824) \\
\hline & Chungcheongnam-do, Gongju-si, Geumgyesan Mt., 29 Apr 2015, Kim Geumgye150150 (KB 521098) \\
\hline & Gangwon-do, Hoengseong-gun, Eoreumgol, 1 May 2014, Kim, Lee \& Kim PH140148 (KB 483757) \\
\hline \multirow[t]{2}{*}{ Papaver dubium $\mathrm{L}$. } & Jeju-do, Seogwipo-si, Daejeong-eup, 21 May 2013, Kim \& Kim kjs130119 (KB 462723) \\
\hline & Jeju-do, Jeju-si, Aewol-eup, Gwakji beach, 28 Apr 2016, Kim, An, Hong \& Park KimJH16007 (KB 563136) \\
\hline P. coreanum Nakai & China, Jilin, Antu, Erdaobaihe, Baekdusan Mt., 7 Jul 2012, Hyun NAPI-2012-0582 (KB 412964) \\
\hline \multirow[t]{3}{*}{ P. rhoeas L. } & Gangwon-do, Sokcho-si, Yeongnangho, 28 May 2014, Kim \& Kim Youngrangho0259 (KB 479526) \\
\hline & Gangwon-do, Gangneung-si, Gyeongpoho, 2 Jul 2014, Kim \& Kim Gyeongpoho0344 (KB 487035) \\
\hline & Chungcheongbuk-do, Chungju-si, Sureuisan Mt., 21 Jun 2015, Lee \& Lee NAPI-P20150388 (KB 533894) \\
\hline \multirow[t]{2}{*}{ P. somniferum $\mathrm{L}$. } & Gyeonggi-do, Gimpo-si, Yangchon-myeon, 26 May 2012, Kim KIMJH12062 (KB 358615) \\
\hline & Jellanam-do, Wando-gun, Cheongsan Island, 28 May 2013, Kim, Kim \& Park KIMJH13137 (KB 434554) \\
\hline
\end{tabular}

Germany). Three DNA barcode regions, specifically chloroplast $r b c L$ and $m a t K$ regions and nuclear ribosomal ITS regions, were amplified via polymerase chain reactions (PCR). Primers for the cpDNA barcodes were published in previous studies (Soltis et al., 1992; Sang et al., 1997; Cuénoud et al., 2002; Kress and Erickson, 2007) and are summarized in Kim et al. (2014). The ITS regions were amplified using the primers its6 and its9 (Youm et al., 2016). For each PCR reaction, $1 \mu \mathrm{L}$ of total DNA was included in a $20 \mu \mathrm{L}$ reaction mixture with Solg EF-Taq DNA polymerase (Solgent, Daejeon, Korea). Amplification of the target regions was conducted with a Veriti thermal cycler (Applied Biosystems, Foster City, CA, USA) under the following conditions: initial denaturation at $95^{\circ} \mathrm{C}$ for $2 \mathrm{~min}, 35$ cycles each at $95^{\circ} \mathrm{C}$ for $30 \mathrm{~s}$, at $55^{\circ} \mathrm{C}$ for $30 \mathrm{~s}$, and at $72^{\circ} \mathrm{C}$ for $1 \mathrm{~min}$, followed by a final extension at $72^{\circ} \mathrm{C}$ for $5 \mathrm{~min}$. PCR products were examined on a $1 \%$ agarose gel in
$1 \mathrm{X}$ TBE buffer, purified, and were sent to Solgent (Daejeon, Korea) or Macrogen (Seoul, Korea) for sequencing, where the sequencing reaction was prepared using the same primers used in PCR with BigDye Terminator v3.1 Cycle Sequencing Kits (Applied Biosystems). Sequences were determined using a 3730xl DNA analyzer (Applied Biosystems). Sequences were edited in Sequencher version 5.0 (Gene Codes Corporation, Ann Arbor, MI, USA), aligned manually.

\section{Phylogenetic analysis}

The chloroplast DNA barcode data were concatenated and analyzed simultaneously. The ITS data were analyzed separately and in combination with the cpDNA data. Dicentra and Corydalis were used as outgroups. Phylogenetic analyses were conducted using the maximum parsimony (MP) method in PAUP* (Swofford, 2002). All characters were treated as 
unordered and were weighted equally in the MP analyses. Gaps resulting from multiple alignments of indels were treated as missing data. Heuristic searches were used with 100 replicates of random sequence additions with tree bisection-reconnection branch swapping, with all of the best trees saved at each step (MulTrees). Bootstrap analyses (Felsenstein, 1985) of 1,000 pseudoreplicates were conducted with heuristic searches, a simple sequence addition in PAUP* to evaluate the support for each clade.

Bayesian phylogenetic analyses (BI) were performed with the program MrBayes version 3.2.5 (Huelsenbeck and Ronquist, 2001). A Markov chain Monte Carlo (MCMC) algorithm was employed for 2,000,000 generations, sampling trees every 100 generations, with four independent chains running simultaneously. Priors were set based on the Akaike information criterion (AIC) using modeltest (Posada and Crandall 1998). The first 4,000 trees (400,000 generations) were discarded as "burn-in," and the remaining trees for which the log-likelihood values had reached a plateau were imported in PAUP* to calculate the posterior probability of each clade.

\section{Hypothesis testing}

A specific phylogenetic hypothesis was tested using the Shimodaira-Hasegawa (1999; SH) test, as implemented in PAUP*. In this analysis, MP trees in extended ITS data in Analysis 2 were generated while constraining the topology using the heuristic search method described previously. Three constraints were tested: (1) forcing Coreanomecon and Hylomecon monophyletic, (2) Coreanomecon and Chelidonium monophyletic, and whether (3) Coreanomecon is a sister to the clade of Chelidonium and Stylophorum. The topology within the Papaver/Meconopsis and Eomecon/Glaucium clades was collapsed in the constraint, leaving no effect on the reconstruction of the MP tree in the given data set. The resultant MP trees with the constrained relationships were evaluated with the original MP trees without the constraint. The objective of the constraint analysis was to test whether each data set does not reject the hypothesis that Coreanomecon is congeneric to Hylomecon or Chelidonium. For the SH test, 10,000 bootstrap replicates were re-sampled using the re-estimated log likelihood (RELL) method.

\section{Assessment of utility of DNA barcodes}

The resolution ability of the species for each DNA barcode and a combinatory DNA barcode were examined based on the percentage of monophyletic species given the MP and BI trees (Kim et al., 2014; Youm et al., 2016). When only one accession in a species was included, it was treated as monophyletic if the branch leading to the accession was greater than zero.

\section{Results}

The final alignment of the cpDNA data ( $r b c L$ and matK) includes 1,371 sites, among which 146 were parsimoniously informative (10.6\%) (Table 1). When outgroups were excluded, 113 sites were informative $(8.2 \%)$. The ITS data consist of 659 sites, of which 221 were parsimoniously informative (33.5\%). For the ingroups, only 191 were informative (29.0\%). The alignment of the combined data of the Korean taxa included 24 sequences with 2,030 sites, among which 367 were

Table 2. Statistics of DNA barcode regions for Papaveroideae in this study. Numerals in parentheses represent comparisons of in-group taxa only. Species resolutions and inter- and intraspecific distances were calculated in the matrices with the Korean taxa only.

\begin{tabular}{cccccc}
\hline \hline & $r b c L+m a t K$ & ITS & $r b c L+$ matK+ ITS & $\begin{array}{c}\text { cpDNA } \\
\text { extended }\end{array}$ & $\begin{array}{c}\text { ITS extended } \\
\text { Number of sequences }\end{array}$ \\
Number of sites & 25 & 24 & 24 & 30 & 63 \\
Number of variable sites & 1,371 & 659 & 2,030 & 1,358 & 753 \\
Number of parsimony-informative sites & $232(137)$ & $303(230)$ & $535(367)$ & $285(204)$ & $442(430)$ \\
Number of MP trees & $146(113)$ & $221(191)$ & $367(304)$ & $167(137)$ & $359(347)$ \\
Tree length & 1 & 4 & 3 & 1 & 42 \\
CI, excluding autapomorphy & 282 & 522 & 810 & 387 & 1,409 \\
RI & 0.8901 & 0.7746 & 0.8026 & 0.7833 & 0.5130 \\
Species resolution power, MP, BI (\%) & 100,100 & 100,100 & 100,100 & - & 0.9294 \\
Intraspecific distance, minimum-maximum & $0.0-0.0022$ & $0.0-0.0317$ & $0.0-0.0100$ & - & - \\
Interspecific distance, minimum-maximum & $0.0769-0.0793$ & $0.3002-0.3199$ & $0.1345-0.1383$ & - & - \\
\hline
\end{tabular}


A. $c p D N A(r b c L+m a t K)$

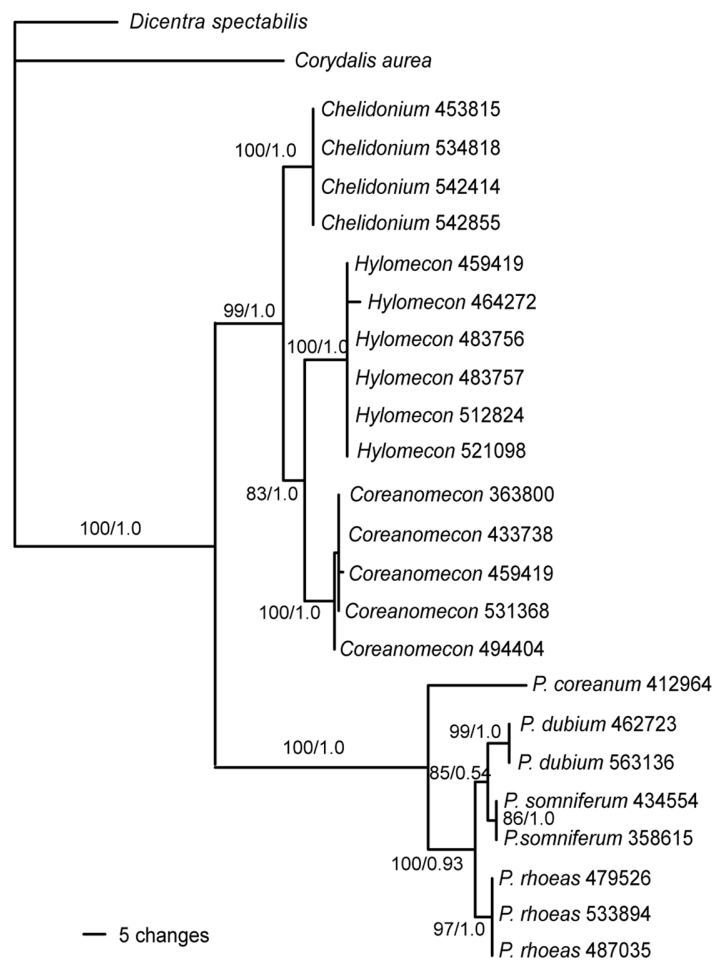

\section{B. ITS}

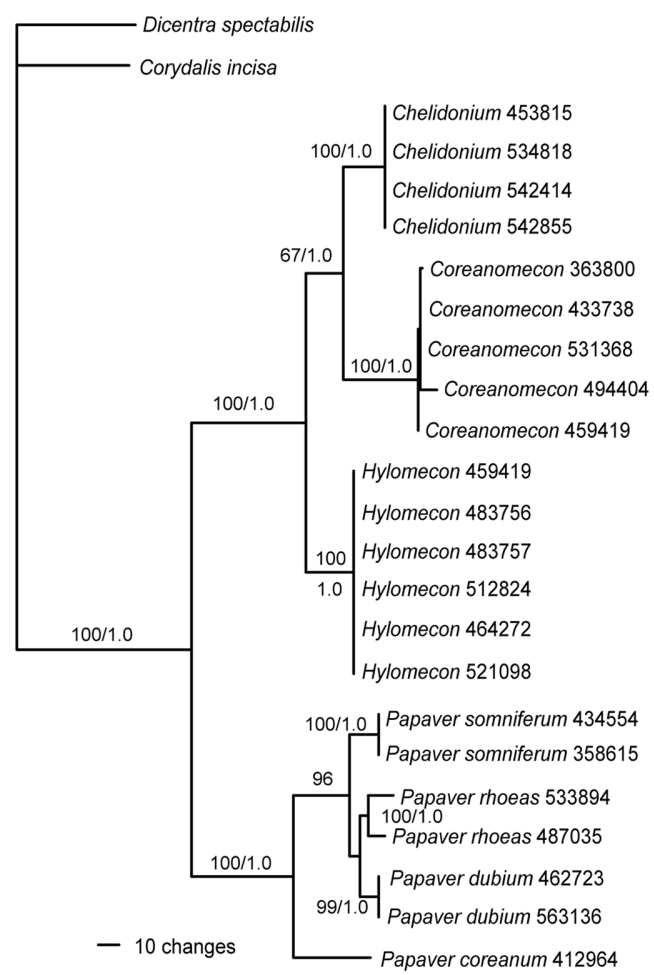

D.

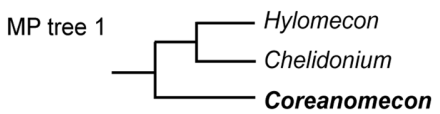

MP tree $2 \& 3$

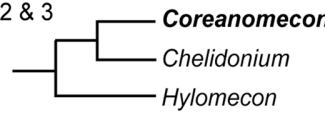

Bayesian tree

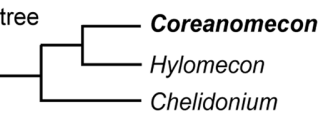

Fig. 1. Phylogenetic analysis of Papaveroideae (Analysis 1). A. A single most parsimonious tree from the analysis of $r b c L$ and $m a t K$ regions; B. One of four maximum parsimony (MP) trees from the analysis of the Internal Transcribed Spacer (ITS) data; C. Strict consensus tree of the combined chloroplast DNA (cpDNA) and ITS data; D, different topologies in the MP analysis and Bayesian analyses of combined data sets. The bootstrap value and Bayesian posterior probability for each clade are indicated on the branch. Numbers following the species name are DNA accession numbers to distinguish each individual. 


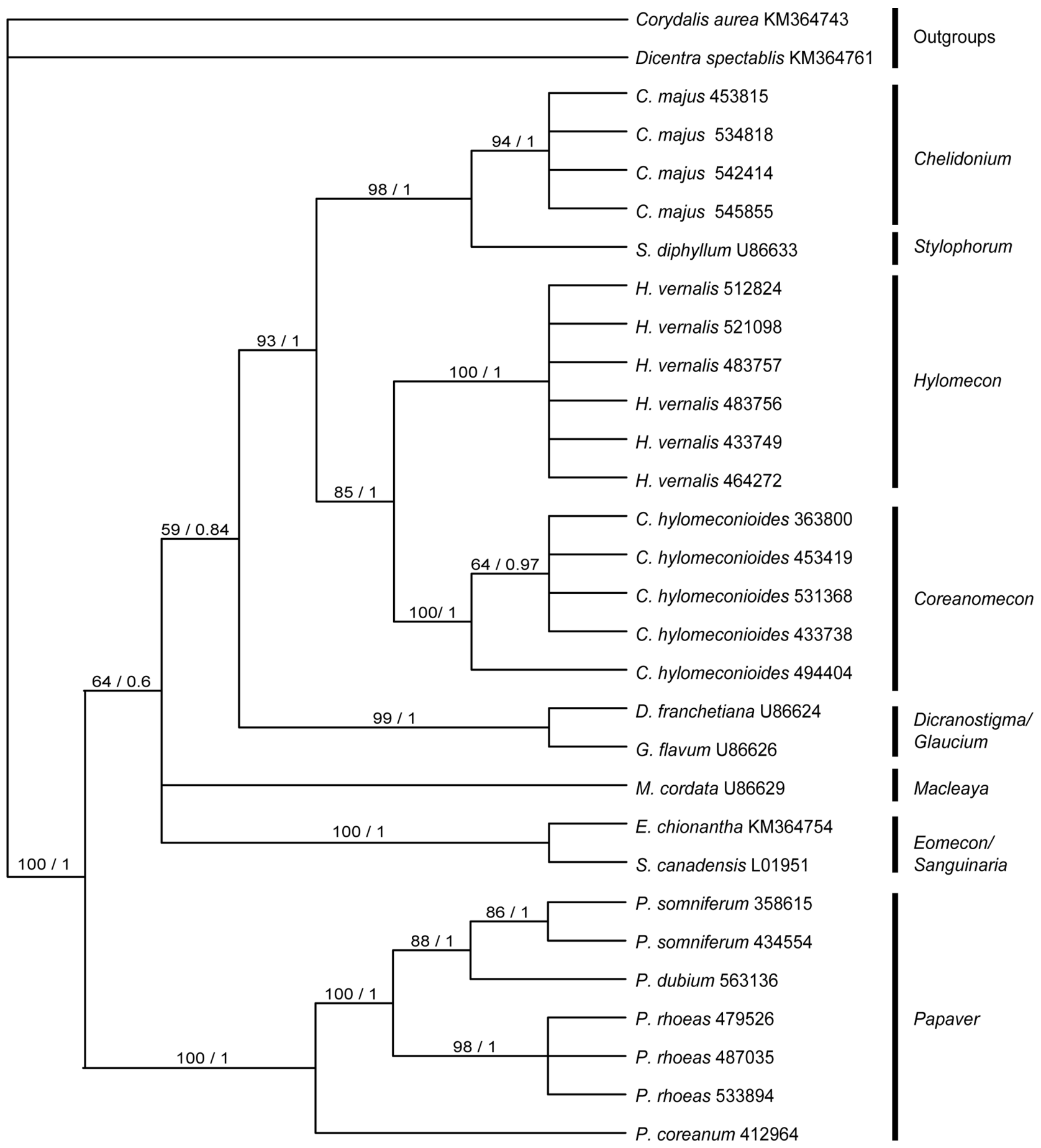

Fig. 2. Strict consensus tree from the phylogenetic analysis of the concatenated $r b c L$ and $m a t K$ data (Analysis 2). The bootstrap value and Bayesian posterior probability for each clade are indicated on the branch. Numbers following the species name are DNA accession numbers to distinguish each individual. Sequences downloaded from GenBank are indicated with the GenBank accession number following the species.

parsimoniously informative. In the extended data of cpDNA and ITS, which had more taxa of Papaveroideae for Analyses 2 and 3, the alignments include 1,358 and 753 sites, respectively, of which $167(12.3 \%)$ and $359(47.7 \%)$ were parsimoniously informative (Table 2)

A phylogenetic analysis of the Korean cpDNA data resolved well-supported clades of Coreanomecon, Chelidonium, Hylomecon, and Papaver (Fig. 1A). Coreanomecon was 


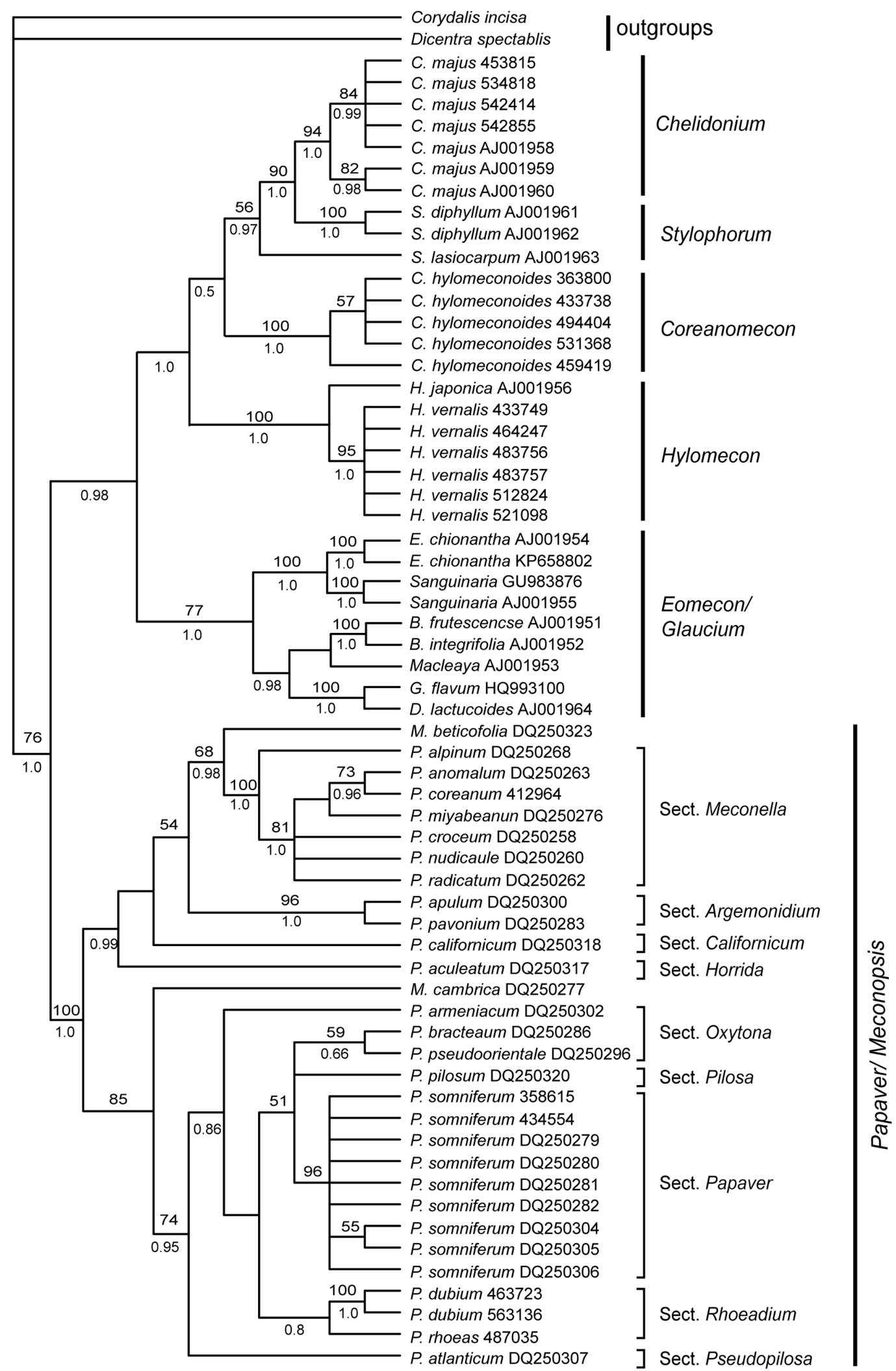

Fig. 3. Strict consensus tree from the phylogenetic analysis of the ITS regions (Analysis 3). The bootstrap value and Bayesian posterior probability for each clade are indicated on the branch. Numbers following the species name are DNA accession numbers to distinguish each individual. Sequences downloaded from GenBank are indicated with the GenBank accession number following the species. 
supported as a sister to Hylomecon. The ITS data also showed that each genus was distinct and well supported (Fig. 1B). However, Coreanomecon is sister to Chelidonium in the nuclear data (Fig. 1B). A maximum parsimony analysis of combined cpDNA and ITS data produced an unresolved relationship regarding Coreanomecon (Fig. 1C). One of the three MP trees placed Coreanomecon as a sister to Hylomecon and Chelidonium and the remaining two trees generate the sister relationship between Coreanomecon and Chelidonium (Fig. 1D). A Bayesian analysis of the combined data supported the contention that Coreanomecon is sister to Hylomecon (Fig. 1D). Within the Papaver clade, P. coreanum is sister to the remaining species, and each species was clearly supported as monophyletic (Fig. 1).

The extended cpDNA data, in which more closely related taxa of Coreanomecon and Papaver were added to gain a better understanding of the phylogenetic relationship of the endemic genus, are consistent with the cpDNA data with Korean taxa only (Fig. 2). Stylophorum, not included in Analysis I (Fig. 1), was sister to Chelidonium, and Coreanomecon was placed to a sister of Hylomecon with strong support. The Glaucium/ Dicranostigma clade was sister to the clade of Chelidonium/ Coreanomecon (Fig. 2). Eomecon and Sanguinaria formed a strongly supported clade. The phylogenetic relationships among Macleaya, the Eomecon and Sanguinaria clade, and the Chelidonium/Coreanomecon clade were unresolved. Monophyly of these genera consisting of the tribe Chelidonieae was weakly supported (Bootstrap support of $64 \%$ and Bayesian post-probability of 0.6) (Fig. 2). Papaver was strongly supported as sister to the tribe Chelidonieae.

Phylogenetic analysis of the extended ITS data showed that Coreanomecon is sister to the clade of Chelidonium and Stylophorum with weak support (Fig. 3). Stylophorum was not supported as monophyletic, as $S$. diphyllum Nutt. is more closely related to Chelidonium than it is to S. lasiocarpus (Oliv.) Fedde. Hylomecon is sister to the clade of Chelidonium, Stylophorum, and Coreanomecon. Other genera in the tribe Chelidonieae, in this case Glaucium, Eomecon, and Dicranostigma, formed a clade with moderate support. Papaver and Meconopsis together formed a strongly supported monophyletic group (Fig. 3).

Three phylogenetic hypotheses (Fig. 4) were tested using the Shimodaira-Hasegawa test. Enforcing a sister relationship of Coreanomecon and Hylomecon (Fig. 4A) in the ITS data, both for Korean taxa only and extended samples, required five additional steps (Table 3). The Shimodaira-Hasegawa test did not reject the alternative topology. Enforcement of the sister relationship of Coreanomecon and Chelidonium (Fig. 4B), as suggested in the ITS data with the Korean taxa only (Fig. 1B), required three additional steps in the cpDNA data with Korean taxa only, and the alternative topology was compatible with the cpDNA data in the S-H test. However, eight additional steps were required to generate the sister relationship of Coreanomecon and Chelidonium in both cpDNA and ITS data with extended taxon samples, and this relationship was significantly rejected in the S-H test. Placement of Coreanomecon as a sister to the clade of Chelidonium and Stylophorum (Fig. 4C) was not available in the data sets with Korean taxa only because Stylophorum is absent in the data. This topology required only three additional steps in the cpDNA data, and the S-H test indicated that it was congruent with the cpDNA data.

The chloroplast $r b c L$ and $m a t K$ data and the ITS data clearly resolved all of the species of Papaveroideae in Korea (Fig. 1). MP and Bayesian analyses of separate and combined data suggested that all of the species in Korea, including the four species of Papaver, are distinguishable according to their DNA barcodes. The resolution power of the DNA barcodes for the
A

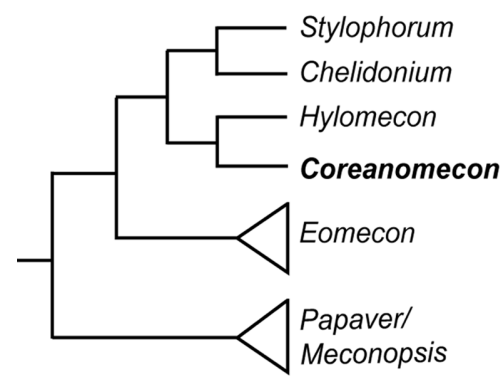

B

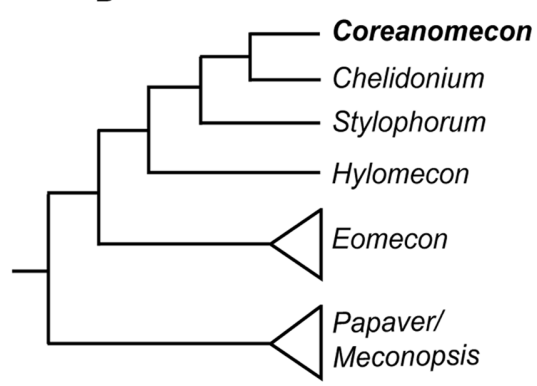

C

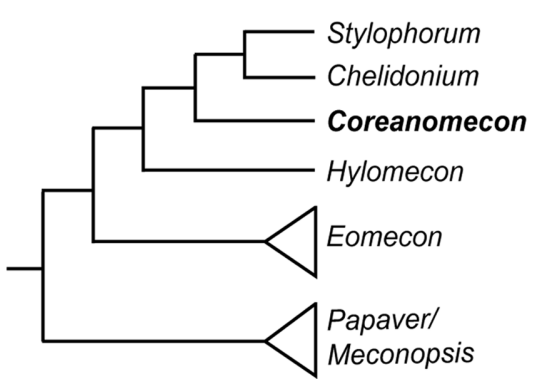

Fig. 4. Schematic diagram of the topologies used in the constraint analyses. Specific relationships within a triangle, not shown in this figure for brevity, were not constrained in the analysis. A. Coreanomecon is forced to be a sister to Hylomecon; B. Coreanomecon is made to be a sister to Chelidonium; C. Coreanomecon is enforced to be a sister to the clade of Stylophorum and Chelidonium. 
Table 3. Tree lengths in the MP analysis, log likelihood scores in the ML analysis, and the significance levels in the Shimodaira-Hasegawa $(\mathrm{S}-\mathrm{H})$ test for constrained topology. Differences in tree lengths and likelihood scores between the best and constrained trees for each data point are indicated in parenthesis. Schematic diagram of constrained topology is shown in Fig. 4

\begin{tabular}{|c|c|c|c|c|}
\hline \multirow{2}{*}{ Data } & \multirow{2}{*}{ Statistics } & \multicolumn{3}{|c|}{ Constrained topology } \\
\hline & & A & B & $\mathrm{C}$ \\
\hline \multirow[t]{3}{*}{ cpDNA (Korean) } & Length (MP) & 282 & $285(+3)$ & Not available \\
\hline & $-\ln (\mathrm{ML})$ & $3,467.365$ & $3,461.414(+5.95)$ & \\
\hline & $p$-value & Best tree & $p>0.05$ & \\
\hline \multirow[t]{3}{*}{ ITS (Korean) } & Length (MP) & $527(+5)$ & 522 & Not available \\
\hline & $-\ln (\mathrm{ML})$ & $5,223.491(+29.94)$ & $5,193.6543$ & \\
\hline & $p$-value & $p>0.05$ & Best tree & \\
\hline \multirow[t]{3}{*}{ cpDNA (extended) } & Length (MP) & 387 & $395(+8)$ & $390(+3)$ \\
\hline & $-\ln (\mathrm{ML})$ & $4,168.107$ & $4,202.249(+34.14)$ & $4,176.214(+8.10)$ \\
\hline & $p$-value & best tree & $p<0.05^{*}$ & $p>0.05$ \\
\hline \multirow[t]{3}{*}{ ITS (extended) } & Length (MP) & $1,414(+5)$ & $1,417(+8)$ & 1,409 \\
\hline & $-\ln (\mathrm{ML})$ & $7,440.479(+2.83)$ & $7,557.902(+20.25)$ & $7,437.650$ \\
\hline & $p$-value & $p>0.05$ & $p<0.05^{*}$ & Best tree \\
\hline
\end{tabular}

Korean Papaveroideae was $100 \%$ (Table 2). The minimum value of the interspecific distances among the species in the cpDNA barcode regions were up to 34 times higher than the maximum value of the intraspecific distance in the three species (Table 2). Similarly, the minimum value of the interspecific distances among the species in the ITS barcode were nine times higher than maximum intraspecific value.

\section{Discussion}

The chloroplast $r b c L$ and $m a t K$ nucleotide sequences and nuclear ITS sequences in a phylogenetic analysis resulted in well-resolved and well-supported phylogenetic trees. Members of Chelidonieae, including the Korean endemic genus Coreanomecon, are strongly supported as monophyletic (Figs. 1-3). In the phylogenetic trees with the Korean taxa only (Fig. 1), all of the species were resolved as distinct lineages. This suggests that the two cpDNA barcode regions and ITS regions are useful to identify incomplete materials that do not bear adequate characters, such as flowers and fruits.

Our phylogenetic analyses of nuclear and chloroplast data show that the position of Coreanomecon is ambiguous. Coreanomecon was consistently placed as a sister to Hylomecon in the cpDNA data (Figs. 1, 2). The nuclear data indicated that it is sister to the Stylophorum and Chelidonium clade (Fig. 3). The constraint analysis results suggest that the two different positions of Coreanomecon (Fig. 4) are not strongly in conflict with each other. The sister relationship between Hylomecon and Coreanomecon represented in the cpDNA data was not rejected in the ITS data by the S-H test (Table 3). Likewise, placement of Coreanomecon as a sister to the clade of Stylophorum and Chelidonium as represented in the extended ITS data was not rejected in the cpDNA data by the S-H test (Table 3 ).

It may be that phylogenetic signals from the cpDNA and ITS data are not enough to resolve these relationships. In the extended cpDNA data, only three additional steps are needed to place Coreanomecon as a sister to the Stylophorum and Chelidonium clade (Table 3). In the extended ITS data, the bootstrap support value for the placement of Coreanomecon as a sister to the Stylophorum and Chelidonium clade is less than $50 \%$, and the posterior probability is very low (Fig. 3). There are five additional steps are required to enforce Coreanomecon as a sister to the Hylomecon (Table 3). Concatenation of data does not help to resolve this issue. Our analysis of combined cpDNA and ITS data generated equivocal topologies (Fig. 1C and D).

Alternatively, the weakly conflicting results from the phylogenetic analysis between the cpDNA and the ITS data may have been associated with hybridization. In this scenario, Coreanomecon may have derived from hybridization between Hylomecon and an ancestor of the Stylophorum and Chelidonium clade. Plastid DNA is shown to be inherited maternally in Chelidonium, Macleaya, and Papaver in Papaveroideae (Zhang et al., 2003). If we assume the maternal inheritance of cpDNA in Hylomecon and Coreanomecon, 
Hylomecon would have been an ovulate parent for Coreanomecon, as the cpDNA trees placed Coreanomecon as a sister to Hylomecon (Fig. 2). The paternal parent would have been a stem lineage of the Stylophorum and Chelidonium clade, as the ITS trees suggest that Coreanomecon is a sister to the clade (Fig. 3). The branch length leading to Coreanomecon is as long as that to Hylomecon (Fig. 1A) or to Chelidonium (Fig. $1 \mathrm{~B})$, suggesting that the hybridization event may be old.

The morphology of Coreanomecon is unique in the tribe Chelidonieae. Coreanomecon has 12-pericolpate pollen, whereas Hylomecon, Chelidonium, Stylophorum lasiocarpum have the 3-colpate type (Lee and Kim, 1984; Kim et al., 1999). Stylophorum diphyllum possesses 12-colpate, rugate, or formaninous pollen (Lee and Kim, 1984). Coreanomecon is the only taxon that has scapose inflorescence in the tribe Chelidonieae. Although a mosaic pattern having both of parental characters or an intermediate form is commonly found in hybrids at early generations, the exhibition of novel characters is common in hybrid taxa at high proportions in angiosperms (Rieseberg and Ellstrand, 1993; Rieseberg et al., 2000). More data are needed for further testing of the origin of Coreanomecon.

The S-H test indicates that a hypothesis of a sister relationship between Coreanomecon and Chelidonium significantly conflicts with both the cpDNA and the ITS data when more taxa are included. The S-H test and phylogenetic analyses of DNA sequence data indicate that inclusion of Coreanomecon in Chelidonium, as was done by Ohwi (1965), is not supported (Table 3). The two genera differ in terms of several morphological characteristics. The leaves of Coreanomecon form a rosette, while those of Chelidonium are attached onto a branched stem. The inflorescence of Coreanomecon is borne from the base of the shoot system and does not have leaves, forming a scape, whereas the inflorescence of Chelidonium is an umbel borne at the terminal of the branch or at the axil of the leaves. Coreanomecon produces orange to red sap and Chelidonium has yellow sap.

Coreanomecon is morphologically similar to Hylomecon; both show similar leaf shapes and sap colors, which lead many authors (Lee, 1973; Park, 1974; Kadereit, 1993) to recognize Coreanomecon as a part of Hylomecon. However, there are significant morphological differences in terms of the inflorescence and pollen type between the taxa, as described above. Our molecular data show that Coreanomecon form a separate clade. Therefore, the morphological and molecular data indicate that Coreanomecon should be recognized as a genus endemic to Korea (Nakai, 1935; Lee, 1980; Kim, 2007; Chung et al., 2017).
The phylogenetic analysis of the extended ITS data show that $P$. coreanum is sister to $P$. anomalum Fedde, belonging to the clade of sect. Meconella (Fig. 3). Members of the sect. Meconella are widely distributed at high latitudes in eastern Asia, Greenland, in northern Canada, and in mountainous regions of Europe and the western North America (Rändel, 1974; Kadereit, 1988). The sect. Meconella is characterized by filiform filaments, pale anthers, and valvately dehiscent capsules (Carolan, 2006). Papaver coreanum match the sectional diagnostic characters well.

The cpDNA and ITS markers provided a high degree of species resolution and resolved all of the species of Papaveroideae in Korea, separately or in combination (Table 2). Adding more taxa (Figs. 2, 3) led to identical results. Considering the economic and forensic importance of Papaveroideae, especially $P$. somniferum, the results of our study are consistent with Lee et al. (2010), showing that DNA barcode regions are highly useful in Papaveroideae.

\section{Acknowledgments}

We are grateful to Jung Won Youm, Yun Gyeong Choi, Dong-Hyuk Lee, and Chae Eun Lim for their help at various stages of this study. We thank the curator and other herbarium staff at KB who generously allowed us to obtain plant samples, as well as two anonymous reviewers for their critical reviews of the manuscript. This work was supported by a research grant, NIBR-201714101, from the National Institute of Biological Resources of Korea.

\section{Conflict of Interest}

The authors declare that there are no conflicts of interest.

\section{Literature Cited}

Carolan, J. C., I. L. I. Hook, M. W. Chase, J. W. Kadereit and T. R. Hodkinson. 2006. Phylogenetics of Papaver and related genera based on DNA sequences from ITS nuclear ribosomal DNA and plastid $t r n \mathrm{~L}$ intron and $t r n \mathrm{~L}-\mathrm{F}$ intergenic spacers. Annals of Botany 98: 141-155.

CBOL Plant Working Group. 2009. A DNA barcode for land plants. Proceedings of the National Academy of Sciences of the United States of America 106: 12794-12797.

Chung, G. Y., K. S. Chang, J.-M. Chung, H. J. Choi, W.-K. Paik and J.-O. Hyun 2017. A checklist of endemic plants on the Korean Peninsula. Korean Journal of Plant Taxonomy 47: 264-288. 
Cronquist, A. 1988. The Evolution and Classification of Flowering Plants. The New York Botanical Garden, Bronx, NY, 555 pp.

Cuénoud, P., V. Savolainen, L. W. Chatrou, M. P. Powell, R. J. Grayer and M. W. Chase. 2002. Molecular phylogenetics of Caryophyllales based on nuclear $18 \mathrm{~S}$ rDNA and plastid $r b c L$, $a t p B$, and $m a t K$ DNA sequences. American Journal of Botany 89: 132-144.

Felsenstein, J. 1985. Confidence limits on phylogenies: an approach using the bootstrap. Evolution 39: 783-791.

Hannan, G. L. and C. Clark. 2011. Papaveraceae. In The Jepson Manual Higher Plants of California II, second edition. Baldwin, B. G., S. Boyd, B. J. Ertter, D. J. Keil, R. W. Patterson, T. J. Rosatti, and D. Wilken (eds.), University of California Press, Berkeley, CA. Pp. 978-986.

Hebert, P. D. N., A. Cywinska, S. L. Ball and J. R. deWaard. 2003. Biological identifications through DNA barcodes. Proceedings of the Royal Society B: Biological Sciences 270: 313321.

Hebert, P. D. N., E. H. Penton, J. M. Burns, D. H. Janzen and W. Hallwachs. 2004. Ten species in one: DNA barcoding reveals cryptic species in the neotropical skipper butterfly Astraptes fulgerator. Proceedings of the National Academy of Sciences of the United States of America 101: 14812-14817.

Hoot, S. B., J. W. Kadereit, F. R. Blattner, K. B. Jork, A. E. Schwarzbach and P. R. Crane. 1997. Data congruence and phylogeny of the Papaveraceae s. 1. based on four data sets: $a t p B$ and $r b c L$ sequences, trnK restriction sites, and morphological characters. Systematic Botany 22: 575-590.

Hoot, S. B., K. M. Wefferling and J. A. Wulff. 2015. Phylogeny and character evolution of Papaveraceae s. 1. (Ranunculales). Systematic Botany 40: 474-488.

Huelsenbeck, J. P. and F. Ronquist. 2001. MRBAYES: Bayesian inference of phylogenetic trees. Bioinformatics 17: 754-755.

Kadereit, J. W. 1988. Sectional affinities and geographical distribution in the genus Papaver L. (Papaveraceae). Beitraräge zur Biologie der Pflanzen 63: 139-156.

Kadereit, J. W. 1993. A revision of Papaver sect. Meconidium. Edinburgh Journal of Botany 50: 125-148.

Kim, H. M., S.-H. Oh, G, S. Bhandari, C.-S. Kim and C.-W. Park. 2014. DNA barcoding of Orchidaceae in Korea. Molecular Ecology Resources 14: 499-507.

Kim, M. 2007. Papaveraceae. In The Genera of Vascular Plants of Korea. Park, C.-W. (ed.), Academy Publishing Co., Seoul. Pp. 216-220.

Kim, M., S.-Y. Kwon and K.-R. Park. 1999. Reexamination the generic status of the Korean endemic Coreanomecon within subfamily Chelidonioideae (Papaveraceae). Korean Journal of
Plant Taxonomy 29: 295-305.

Kress, W. J. and D. L. Erickson. 2007. A two-locus global DNA barcode for land plants: the coding $r b c \mathrm{~L}$ gene complements the non-coding $t r n \mathrm{H}-p s b \mathrm{~A}$ spacer region. PLoS ONE 2: e508.

Kress, W. J., K. J. Wurdack, E. A. Zimmer, L. A. Weigt and D. H. Janzen. 2005. Use of DNA barcodes to identify flowering plants. Proceedings of the National Academy of Sciences of the United States of America 102: 8369-8374.

Lahaye, R, M. van der Bank, D. Bogarin, J. Warner, F. Pupulin, G. Gigot, O. Maurin, S. Duthoit, T. G. Barraclough and V. Savolainen. 2008. DNA barcoding the floras of biodiversity hotspots. Proceedings of the National Academy of Sciences of the United States of America 105: 2923-2928.

Lee, E. J., I. K. Hwang, N. Y. Km, K. L. Lee, M. S. Han, Y. H. Lee, M. Y. Kim and M. S. Yang. 2010. An assessment of the utility of universal and specific genetic markers for opium poppy identification. Journal of Forensic Sciences 55: 12021208.

Lee, S. and M. Y. Kim. 1984. A palynotaxonomic study of Coreanomecon hylomecoides Nakai (Papaveraceae) and its closely related species. Korean Journal of Plant Taxonomy 14: 181186.

Lee, Y. N. 1973. Taxonomic study on genus Hylomecon. Journal of Korean Research Institute of Better Living 11: 127-136. (in Korean)

Lee, T. B. 1980. Illustrated Flora of Korea. Hyangmunsa, Seoul, 990 pp.

Mabberley, D. J. 2008. Mabberley's Plant-Book: A Portable Dictionary of the Vascular Plants. 2nd ed. Cambridge University Press, Cambridge, NY, 858 pp.

Nakai, T. 1935. Coreanomecon hylomecoides Nakai. Journal of Japanese Botany 11: 151-152.

Ohwi, J. 1965. Flora of Japan. Smithsonian Institution, Washington, D.C., 1067 pp.

Park, M. K. 1974. Keys to the Herbaceous Plants in Korea. Chungeumsa, Seoul, 593 pp.

Posada, D. and K. A. Crandall. 1998. Modeltest: testing the model of DNA substitution. Bioinformatics (Oxford) 14: 817-818.

Rändel U. 1974. Beitrage zur Kenntnis der Sippenstruktuir der Gattung Papaver L. Sectio Scapiflora Reihenb. Im vergleich mit P. alpinum L. (Papaveraceae). Feddes Repert 86: 19-37.

Rieseberg, L. H. and N. C. Ellstrand. 1993. What can molecular and morphological markers tell us about plant hybridization? Critical Reviews in Plant Sciences 12: 213-241.

Rieseberg, L. H., S. J. Baird and K. A. Gardner. 2000. Hybridization, introgression, and linkage evolution. Plant Molecular Biology 42: 205-224.

Sang, T., D. J. Crawford and T. Stuessy. 1997. Chloroplast DNA 
phylogeny, reticulate evolution, and biogeography of Paeonia (Paeoniaceae). American Journal of Botany 84: 1120-1136.

Simpson, M. G. 2010. Plant Systematics. 2nd ed. Elsevier, Amsterdam, $740 \mathrm{pp}$.

Soltis, P. S., D. E. Soltis and C. J. Smiley. 1992. An $r b c L$ sequence from a Miocene Taxodium (bald cypress). Proceedings of the National Academy of Sciences of the United States of America 89: 449-451.

Son, S.-W., J.-M. Chung, J.-K. Shin, B.-C. Lee, K.-W. Park and S. J. Park. 2012. Distribution, vegetation characteristics and assessment of the conservation status of a rare and endemic plant, Coreanomecon hylomeconoides Nakai. Korean Journal of Plant Taxonomy 42: 116-125.

Swofford, D. L. 2002. PAUP* Phylogenetic analysis using parsimony (* and other methods), version 4.0. Sinauer Associates, Sunderland, MA.

Takhtajan, A. 1997. Diversity and Classification of Flowering Plants. Columbia University Press, New York, 643 pp.

The Angiosperm Phylogeny Group. 2003. An update of the Angiosperm Phylogeny Group classification for the orders and families of flowering plants: APG II. Botanical Journal of the Linnean Society 141: 399-436.

The Angiosperm Phylogeny Group. 2009. An update of the Angiosperm Phylogeny Group classification for the orders and families of flowering plants: APG III. Botanical Journal of the Linnean Society 161: 105-121.

The Angiosperm Phylogeny Group. 2016. An update of the Angiosperm Phylogeny Group classification for the orders and families of flowering plants: APG IV. Botanical Journal of the
Linnean Society, 181: 1-20.

Thorne, R. F. 1992. Classification and geography of the flowering plants. Botanical Review 58: 225-348.

Tripathi, A. M., A. Tyagi, A. Kumar, A. Singh, S. Singh, L. B. Chaudhary and S. Roy. 2013. The internal transcribed spacer (ITS) region and $t r n \mathrm{H}-p s b \mathrm{~A}$ are suitable candidate loci for DNA barcoding of tropical tree species of India. PLoS ONE 8: e57934.

Wang, W., A.-M. Lu, Y. Ren, M. E. Endress and Z.-D. Chen. 2009. Phylogeny and classification of Ranunculales: evidence from four molecular loci and morphological data. Perspectives in Plant Ecology, Evolution and Systematics 11: 81-110.

Xiang, X.-G., H. Hu, W. Wang and X.-H. Jin. 2011. DNA barcoding of the recently evolved genus Holcoglossum (Orchidaceae: Aeridinae): a test of DNA barcode candidates. Molecular Ecology Resources 11: 1012-1021.

Youm, J. W., S.-W. Han, S. W. Seo, C. U. Lim and S.-H. Oh. 2016. DNA barcoding of Schisandraceae in Korea. Korean Journal of Plant Taxonomy 46: 273-282.

Zhang, M., Z. Su, M. Lidén and C. Grey-Wilson. 2008. Papaveraceae. In Flora of China. Vol. 7. Menispermaceae through Capparaceae. Wu, Z. Y., P. H. Raven and D. Y. Hong (eds.), Science Press, Beijing and Missouri Botanical Garden Press, St. Louis, MO. Pp. 261-293.

Zhang, Q., Y. Liu and Sodmergen. 2003. Examination of the cytoplasmic DNA in male reproductive cells to determine the potential for cytoplasmic inheritance in 295 angiosperm species. Plant and Cell Physiology 44: 941-951. 